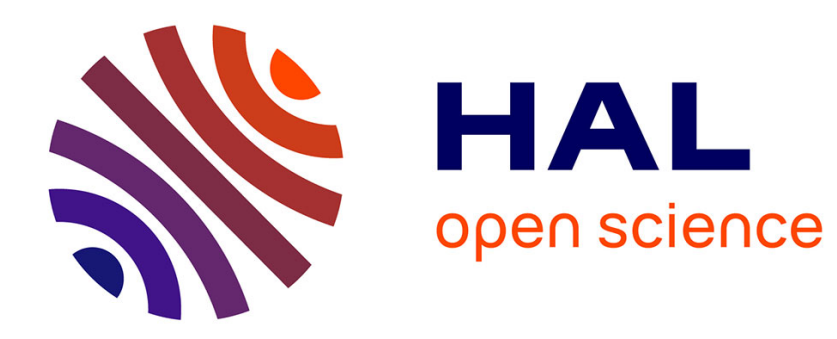

\title{
Eye Position Specificity of Saccadic Adaptation
}

\author{
Nadia Alahyane, Denis Pelisson
}

\section{To cite this version:}

Nadia Alahyane, Denis Pelisson. Eye Position Specificity of Saccadic Adaptation. Investigative Ophthalmology \& Visual Science, 2004, 45 (1), pp.123-130. 10.1167/iovs.03-0570 . hal-02197092

\section{HAL Id: hal-02197092 \\ https://hal.science/hal-02197092}

Submitted on 30 Jul 2019

HAL is a multi-disciplinary open access archive for the deposit and dissemination of scientific research documents, whether they are published or not. The documents may come from teaching and research institutions in France or abroad, or from public or private research centers.
L'archive ouverte pluridisciplinaire HAL, est destinée au dépôt et à la diffusion de documents scientifiques de niveau recherche, publiés ou non, émanant des établissements d'enseignement et de recherche français ou étrangers, des laboratoires publics ou privés. 


\title{
Eye Position Specificity of Saccadic Adaptation
}

\author{
Nadia Alabyane and Denis Pélisson
}

Purpose. The accuracy of saccadic eye movements is maintained throughout life by adaptive mechanisms. With the double-step target paradigm, eight human subjects were investigated to determine whether saccadic adaptation depends only on the eye-displacement vector, or also on eye position as a context cue when two saccades of identical vector are adapted simultaneously.

Methods. First, bidirectional adaptations (BDAs) of horizontal saccades of the same vector were induced in a single training phase. Each direction of adaptation in BDAs (backward and forward) was linked to one vertical eye position (e.g., forward adaptation performed with the eyes directed $12.5^{\circ}$ upward and backward adaptation with the eyes $25^{\circ}$ downward) and alternated from trial to trial. Second, unidirectional adaptations (UDAs) were tested in two control conditions in which training trials of a single direction (backward or forward) were presented at both $12.5^{\circ}$ and $-25^{\circ}$ eye elevations.

RESUlts. Opposite changes in saccade amplitude could develop simultaneously in BDA, indicating that saccadic adaptation depends on orbital eye position. Comparing these data with the control conditions further indicated that eye position specificity was complete for backward, but not for forward, adaptation.

Conclusions. The results indicate that saccadic adaptation mechanisms use vectorial eye displacement signals, but can also take eye position signals into account as a contextual cue when the training involves conflicting saccade amplitude changes. (Invest Ophthalmol Vis Sci. 2004;45:123-130) DOI: 10.1167/iovs.03-0570

Saccades are fast eye movements that allow accurate fove$\checkmark$ ation of selected objects. The saccadic system includes adaptive mechanisms that maintain saccade accuracy throughout life. Saccadic adaptation can be experimentally induced by applying the double-step target paradigm pioneered by McLaughlin $^{1}$ in which the target is systematically displaced during the saccadic response, eliciting a postsaccadic visual error without any conscious perception of target displacement (saccadic suppression phenomenon). Applying systematically backward or forward (relative to the initial target step) intrasaccadic target displacement over successive trials leads to a progressive reduction or increase of saccade amplitude and hence to a gradual reduction of the postsaccadic visual error.

From the Institut de la Santé et de la Recherche Médicale (INSERM) Unité 534, Espace et Action, IFR19 Institut Fédératif des Neurosciences de Lyon, Bron, France.

Supported by Institut de la Santé et de la Recherche Médicale, Unité 534

Submitted for publication June 6, 2003; revised September 2, 2003; accepted September 8, 2003

Disclosure: N. Alahyane, None; D. Pélisson, None

The publication costs of this article were defrayed in part by page charge payment. This article must therefore be marked "advertisement" in accordance with 18 U.S.C. $\$ 1734$ solely to indicate this fact.

Corresponding author: Denis Pélisson, INSERM Unité 534, Espace et Action, 16 avenue du doyen Lépine, 69500 Bron, France; pelisson@lyon.inserm.fr.
We now describe two main properties of saccadic adaptation that studies using this double-step paradigm have revealed.

On the one hand, it was found that saccadic adaptation in humans $^{2-5}$ as well as in monkeys ${ }^{6-8}$ is specific to the amplitude and the direction of the trained saccade. Indeed when a given saccade is adapted, all saccades of the same amplitude and direction (i.e., same vector)-regardless of their starting orbital position-show a similar adaptive modification. Conversely, saccades of a different displacement vector demonstrate less or no change.

On the other hand, more recent studies began to provide evidence that adaptation depends not only on the saccade vector but also on various contexts (see the Discussion section). One of these contexts, orbital eye position, is particularly interesting, because in everyday life our eyes saccade from constantly changing orbital positions. Shelhamer and Clendaniel $^{9}$ investigated whether eye position can serve as a contextual cue for saccadic adaptation. In their study, two different types of adaptation (forward and backward) were elicited concurrently in a single session and associated with different eye positions. Their results suggested that both horizontal and vertical eye positions could provide a context for the adaptation of horizontal saccades allowing the saccadic system to switch between a state of increased saccade amplitude for one eye position and a state of decreased amplitude for another eye position. However, this study ${ }^{9}$ had the following limitations: (1) the number of tested subjects (four) is rather small when one considers the well-known intersubject variability in saccadic adaptation, (2) the efficacy of context-specific adaptations was evaluated in only one of the four subjects and, in addition, this evaluation was performed in a control experiment that only indirectly tested possible interferences between the increased and the decreased saccade adaptations, and (3) the two different types of adaptations were not presented randomly in interleaved trials but in alternating blocks of 20 identical trials.

The present work, performed in parallel with the previous study ${ }^{9}$ with the same objective, contains several original points allowing us to circumvent the limitations of the prior study. In eight subjects we elicited both backward and forward adaptations of horizontal saccades in a single training session, each direction being related to a different eye elevation (either $12.5^{\circ}$ up or $25^{\circ}$ down) and alternating randomly from one trial to the next. In addition, we compared this bidirectional adaptation (BDA) condition with two unidirectional adaptation (UDA) conditions (control conditions), in which backward or forward intrasaccadic steps were identical, with the eyes looking up and down. Some of the results have been submitted for publication in abstract form. ${ }^{10}$

\section{Materials ANd Methods}

\section{Subjects}

Eight voluntary subjects (five women, three men; age range, 19-42 years) took part in this study. Two subjects used their normal corrective lenses during all experiments. Five subjects were aware of the global purpose of the study whereas the other three were totally naive. This work complied with the Declaration of Helsinki. 


\section{EXPERIMENTAL SET UP}

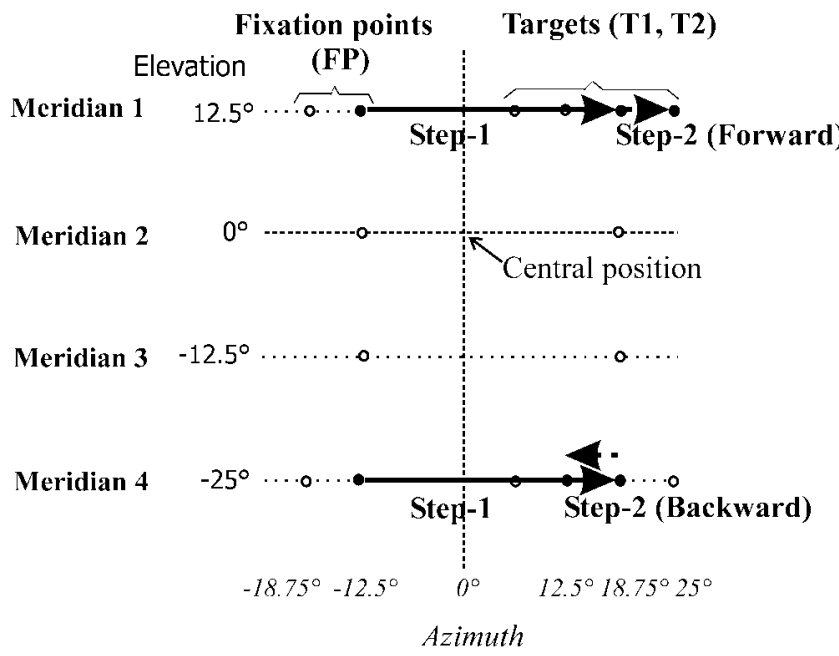

Figure 1. Experimental setup. LEDs, situated on a spherical board centered on the subject's cyclopean eye, were illuminated on horizontal meridians 1 to 4 (elevations of $12.5^{\circ}, 0^{\circ},-12.5^{\circ}$, and $-25^{\circ}$ ). Two fixation points (FPs) at an azimuth of $-18.75^{\circ}$ and $-12.5^{\circ}$ were used on meridians 1 and 4 in adaptation sessions and a single FP at $-12.5^{\circ}$ was used on all four meridians in test sessions. In all sessions, the primary saccade was elicited by a rightward target displacement from FP to T1 (step $1=31.25^{\circ}$ ); during adaptation sessions, a second target displacement from $\mathrm{T} 1$ to $\mathrm{T} 2$ ( primary saccade. Filled circles connected by arrows illustrate one example of double stimulations (step 1 followed by step 2) used during bidirectional adaptations, with forward and backward adaptations induced along meridians 1 and 4, respectively. FP, fixation point; T1, target-1; T2, target-2.

\section{Experimental Setup}

The experimental set up is shown in schematic form in Figure 1. The subject sat in front of a concave spherical target board so that the center (intersecting point of the two dashed lines) was on the subject's naso-occipital axis at $110 \mathrm{~cm}$ (sphere radius) from the cyclopean eye. Red light-emitting diodes (LEDs), used as visual targets, were placed in holes made in the sphere according to a Cartesian squaring. For the present study, visual targets were located along four horizontal meridians at an elevation of $12.5^{\circ}, 0^{\circ},-12.5^{\circ}$, and $-25^{\circ}$ relative to the sphere center (meridians 1-4, respectively). The experiments were performed in dim light so that the subject saw only the illuminated target. The subject kept his head motionless by biting on a dental impression.

\section{Experimental Protocol}

The protocol consisted of three experimental conditions, each subdivided into three sequential sessions: pretest ( 40 trials), adaptation (240 trials), and posttest (40 trials) sessions. The pre- and posttest sessions were always identical, whereas the type of adaptation session differed between the three experimental conditions: BDA, unidirectional backward (UDBA) or unidirectional forward (UDFA). All subjects performed the three experimental conditions on separate days to avoid interactions between them: the BDA condition was always performed first, followed by the UDBA and UDFA conditions performed in random order across subjects.

Adaptation Procedure. At the beginning of a trial, the subject fixated an LED (fixation point; FP) located in the left hemifield at a distance of $12.5^{\circ}$ from the center. After a delay of $1200 \mathrm{~ms}$, the FP was turned off while another target (T1) was simultaneously illuminated in the right hemifield at an $18.75^{\circ}$ location, corresponding to a target displacement of $31.25^{\circ}$ (step 1). When the velocity of the saccade toward T1 exceeded a threshold, variable according to subjects (100 $\mathrm{deg} / \mathrm{s}$ on average), T1 was replaced by another target (T2) located $6.25^{\circ}$ away (step 2 : amplitude $=20 \%$ of step 1 ). Step 2 was backward with respect to step 1 in amplitude-reducing adaptation (Fig. 2B) and forward in amplitude-increasing adaptation (Fig. 2D). A second FP located at $-18.75^{\circ}$ in the left hemifield was also used in conjunction with a target $\mathrm{T} 1$ at $12.5^{\circ}$ in the right hemifield. The presentation of the two $\mathrm{FP} / \mathrm{T} 1$ pairs $\left(-12.5^{\circ} / 18.75^{\circ}\right.$ or $\left.-18.75^{\circ} / 12.5^{\circ}\right)$ was randomized to increase the level of uncertainty in the sequence of trials and to discourage anticipatory behavior. Each trial lasted $1800 \mathrm{~ms}$ with an intertrial period (duration of FP presentation) of $1200 \mathrm{~ms}$. Six blocks of 40 trials were presented, and the subject could take a 1 to 2 minutes' rest between the successive blocks to avoid fatigue and loss of concentration.

BDA Condition. In this condition, adaptation to backward and forward target steps were elicited concurrently and associated with different horizontal meridians. For four subjects, target step 2 was forward when the subject's eyes were directed at meridian 1 and backward when the subject's eyes were directed at meridian 4 , as illustrated in Figure 1. For the other four subjects, step 2 was backward along meridian 1 and forward along meridian 4, as illustrated in Figure 2. The position of FP and the associated direction of step 2 were randomized from trial to trial.

UDA Conditions. These two conditions served as controls for the BDA condition and were both characterized by the same step 2 occurring along meridians 1 and 4 . In the UDBA a leftward step 2 was used to reduce saccade amplitude and in the UDFA a rightward step 2 was used to increase saccade amplitude.

Pretest and Posttest Sessions. Single target steps from FP $\left(-12.5^{\circ}\right)$ to $\mathrm{T} 1\left(18.75^{\circ}\right)$ preceded (pretest) and followed (posttest) each adaptation session (see Figs. 2A, 2C). In these test sessions, the target $\mathrm{T} 1$ did not jump to a secondary position. Instead, T1 was extinguished at the beginning of the saccade to prevent any postsaccadic fixation error from modifying saccade amplitude in subsequent trials through deadaptation mechanisms. The same target displacements as in the adaptation sessions were performed on horizontal meridians 1 and 4 . In addition, single steps were performed on meridians 2 and 3 (elevation of $0^{\circ}$ and $-12.5^{\circ}$, respectively) to test the existence of a transfer of adaptation to nontrained saccades executed along these two intermediate meridians (data not shown in this article). Each test session, therefore, contained four different types of stimulations (one per meridian) repeated randomly 10 times.

\section{Eye Movement Recording and On-line Signal Processing}

Horizontal and vertical eye movements were measured by means of an infrared system (Eye-link; SMI, Berlin, Germany). Immediately before the start of the session in each experimental condition, a calibration was performed using a rectangular array of nine targets that contained the zone of the oculomotor field explored during the recording sessions. Target (LED) presentation and data collection were controlled by a computer program (DataWave, Longmont, CO). Horizontal and vertical positions of each eye were displayed and recorded on disc for off-line analysis (sampling rate $500 \mathrm{~Hz}$ ). To detect saccades on-line, a signal of angular position of the left eye was processed by an electronic circuit (low-pass filtering, differentiation, and comparison with an adjustable threshold). The output of this circuit was used by the computer to trigger the intrasaccadic target displacement during the adaptation sessions and to switch the target off in the pre- and posttest sessions.

\section{Off-line Data Analysis}

Extraction of Parameters. The horizontal component of movements of the left eye was analyzed off-line. After filtering (finite 


\section{INDIVIDUAL RESPONSES (BDA CONDITION)}

Backward (upper meridian: 1)

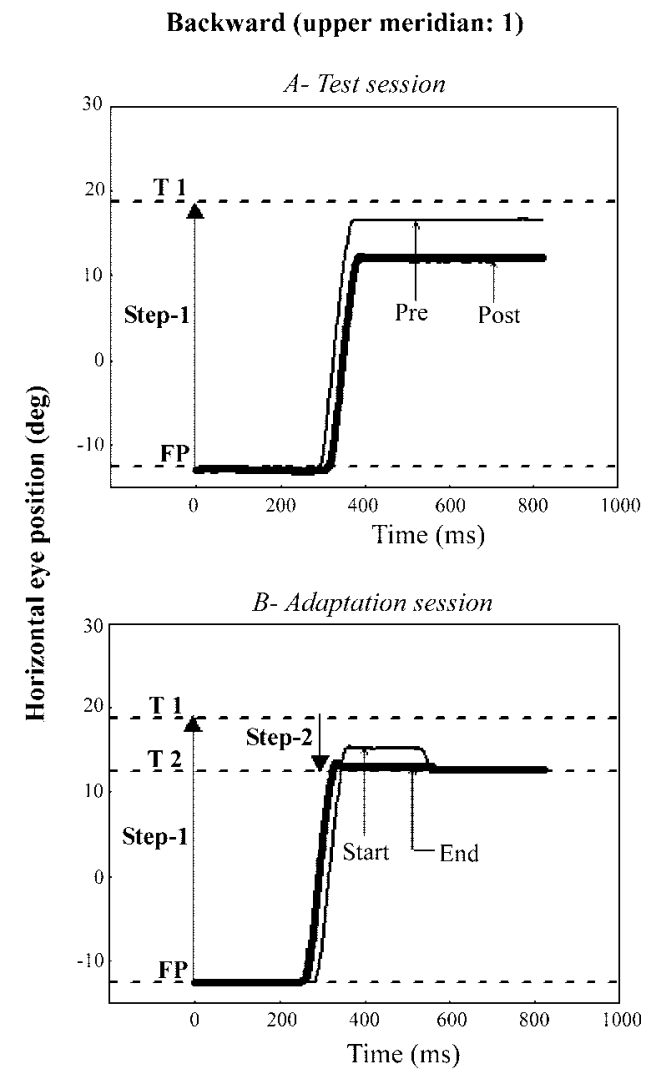

Forward (lower meridian: 4)

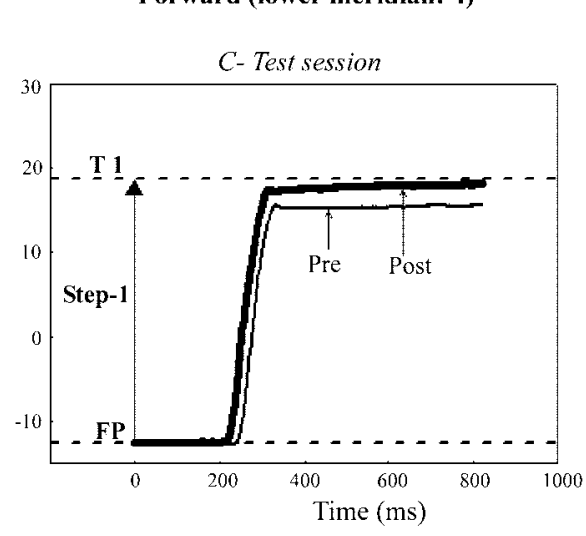

D-Adaptation session

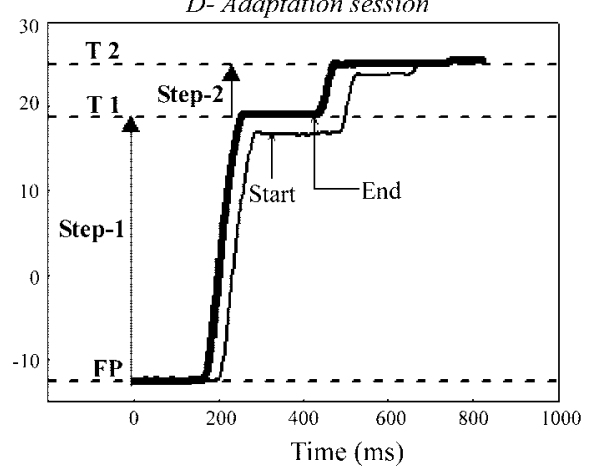

Figure 2. Examples of saccadic responses recorded in subject $\mathrm{F}$ before, during, and after bidirectional adaptation (BDA condition). A first target displacement (step 1) elicited a primary saccade, during which T1 was turned off in test sessions or displaced (step 2) in the adaptation session. Step 2 was backward in uplooking trials (B) and forward in down-looking trials (D). (A, C) Saccadic responses in the test sessions preceding (thin line: Pre) or after (thick line: Post) the adaptation session. (B, D) Saccades recorded during the first (thin line) and the last (thick line) block of adaptation trials. FP, fixation point; T1, target-1; T2, target-2.

impulse response [FIR] filter with a $70-\mathrm{Hz}$ cutoff frequency, residual noise level, $<0.5 \mathrm{deg}$ ), the detection of the start and the end of the saccade was made on the basis of a velocity threshold set at $40 \mathrm{deg} / \mathrm{s}$. Each primary saccade was checked individually by the experimenter who could manually correct the start and end-point markings if necessary. Trials in which an eye blink occurred during the primary saccade and in which saccades had not been correctly detected online were eliminated. Saccades with amplitude less than $21.9^{\circ}$ or more than $39.1^{\circ}$ or with latency less than $80 \mathrm{~ms}$ were also excluded from the analysis. On the whole, eliminated trials represented a proportion of $3.6 \% \pm 3.7 \%$. The horizontal amplitude of each primary saccade was calculated as the difference between initial and final eye positions. The percent amplitude change of the primary saccade between the posttest and the pretest sessions was calculated as follows: amplitude change $=$ [(posttest mean amplitude - pretest mean amplitude)/pretest mean amplitude] $\times 100$.

Statistical Analysis. Adaptation Level. The mean amplitude of saccades recorded in the test sessions was submitted to two different two-way repeated measures analyses of variance (ANOVAs), the first testing the effectiveness of the BDA protocol to induce oppositely directed amplitude changes, and the second comparing separately for forward and backward adaptations the efficacy of the BDA and the UDA protocols. A third two-way, repeated-measures ANOVA was applied to the absolute difference between the pre- and posttest mean amplitudes of saccades obtained in UDA conditions to compare the efficacy of UDBA versus UDFA. The significance level for all ANOVAs was set at $P<0.05$, and Fisher least-significant difference [LSD] tests were used for post hoc comparisons.

Adaptation Time Course. When evaluating the temporal development of adaptation, we found that the shape of the relationship between saccade amplitude and number of trials varied between subjects, but often no clear asymptotic level was detected. Therefore, an exponential fitting of the adaptation time course ${ }^{2,11}$ was not adequate for our data, and a linear regression method was used instead. This analysis revealed that in five subjects, the slope of the relationship was significant $(P<0.05)$ in all three conditions (BDA, UDBA, UDFA). In these subjects, the absolute slope values were submitted to a two-way, repeated-measures ANOVA with the factors adaptation type and adaptation direction.

\section{Results}

\section{Adaptation Level}

BDA Condition. General Features. Figure 2 illustrates representative saccadic responses of subject $F$ who was submitted, during a single training session, to backward adaptation trials in which his eyes were directed upward (meridian 1), interleaved with forward adaptation trials in which his eyes were directed downward (meridian 4).

Backward Adaptation. Before adaptation (pretest), the subject performed primary saccades landing very close to T1. At the start of the adaptation session (first block of trials), the introduction of step 2 led the subject to overshoot T2 and to produce a corrective saccade redirecting the eyes back to T2. Toward the end of the adaptation session (last block of trials), however, both saccades decreased, and notably the primary saccade now landed closer to T2. After adaptation (posttest), the saccade amplitude was smaller than before adaptation.

Forward Adaptation. The introduction of step 2 at the beginning of adaptation caused the primary saccade, in this case, to undershoot the target, requiring the execution of two forward corrective saccades. By the end of adaptation, the primary saccade was larger and was followed only by one 
Backward vs Forward

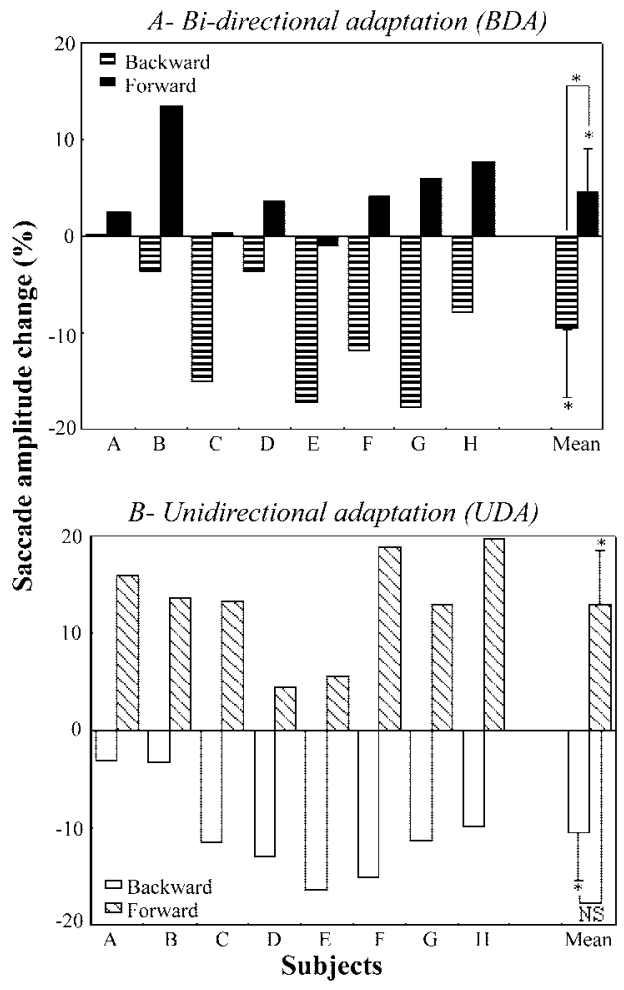

Bi- (BDA) vs Uni- (UDA) directional
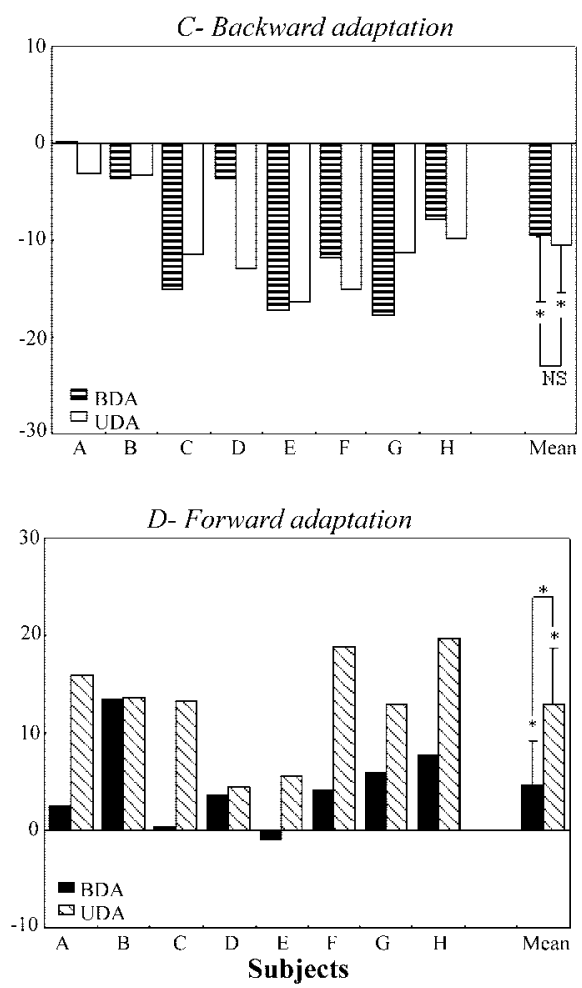

Figure 3. Changes of primary saccade amplitude between the posttest and the pretest sessions in all subjects $(\mathrm{A}-\mathrm{H})$ and averaged across subjects (Mean). (A, B) Effect of backward versus forward adaptation in the BDA (A) and UDA (B) condition. (C, D) Comparison of BDA versus UDA condition for backward (C) and forward (D) adaptation. Asterisks near each of the last two bars indicate statistically significant differences $(P<0.05)$ of mean saccade amplitude between pretest and posttest sessions, and asterisks between the last two bars indicate statistically significant differences between the two conditions depicted in each panel (ANOVA); NS, no significant difference $(P>0.05)$. Error bars, SD. corrective saccade. In the posttest session, the saccade amplitude was larger than before adaptation.

These individual responses suggest that the posttest saccadic amplitude was reduced relative to the pretest for the backward adaptation and was increased for the forward adaptation, when opposite-direction adaptation trials were interleaved randomly during a single BDA session.

Statistical Analysis of Adaptation Level. As can be seen in Figure $3 \mathrm{~A}$, the changes of saccade amplitude between the posttest and the pretest were consistent with the direction of step 2, except for subjects A (backward) and E (forward). Backward adaptation seemed stronger than forward adaptation for all subjects but subject B. Indeed, saccade amplitude was reduced on average by $9.6 \% \pm 6.9 \%$ after backward adaptation, whereas it increased by $4.6 \% \pm 4.6 \%$ after forward adaptation. The two-way ANOVA (adaptation direction $\times$ type of test session) applied to the mean amplitude of saccades recorded in the test sessions showed a significant difference between backward adaptation and forward adaptation $(P<0.01)$, but no significant difference between the two types of test (pretest and posttest) sessions $(P=0.2)$. The independent-factor vertical eye position had no significant effect $(P=0.6)$. However, a significant interaction (adaptation direction $\times$ type of test session) was found $(P<0.001)$. Posthoc tests showed that the only nonsignificant $(P=0.2)$ difference in mean amplitude was between the two pretests. In conclusion, BDA induced simultaneously in the same session led to saccade amplitude modifications in the direction specified by step 2 . This was valid, irrespective of the meridian ( 1 or 4 ) along which backward or forward adaptation was induced.

BDA Versus UDA. To find out whether BDAs were as effective as control adaptations (UDAs), we compared these two situations separately for backward and forward adapta- tions. Figure $3 \mathrm{C}$ shows that backward adaptations induced similar saccade amplitude changes in the BDA and UDA conditions (mean amplitude change of approximately $-12 \%$ ). In contrast, the forward adaptation (Fig. 3D) was more effective in the UDA condition (mean amplitude change: $13 \% \pm 5.6 \%$ ) than in the BDA condition (mean amplitude change: $4.6 \% \pm$ $4.6 \%$ ), except for subjects $\mathrm{B}$ and $\mathrm{D}$ who showed equivalent forward adaptations in the two conditions.

These results were confirmed by the two-way ANOVAs (adaptation condition $\times$ type of test session) which were applied separately for backward and forward adaptations. For backward adaptation, there was a significant difference between the two types of test sessions $(P<0.01)$ but not between the two types of adaptations $(P=0.2)$ and no interaction $(P=0.8)$. For forward adaptation, there was a significant difference between the two types of test sessions $(P<$ $0.01)$ but not between the two types of forward adaptations $(P=0.1)$ and a significant interaction $(P<0.01)$. Post hoc tests showed that the only nonsignificant $(P=0.4)$ difference of mean amplitude was between the two pretests. Finally, for both backward and forward adaptations, the vertical eye position included as an independent factor in the ANOVA had no significant effect $(P=0.3$ and $P=0.5$, respectively). This suggests that the pattern of results was valid, irrespective of the meridian along which subjects actually experienced backward or forward adaptation in the BDA condition.

We also compared backward and forward adaptations in the UDA conditions (Fig. 3B). On average, these two types of separately induced adaptations were similar and resulted in a mean change of saccade amplitude of approximately $12 \%$. Indeed, the two-way ANOVA applied to the absolute difference of mean saccade amplitude between the post- and the pretest revealed no significant difference between the two adaptation 


\section{SACCADE AMPLITUDE CHANGE DURING BDA}

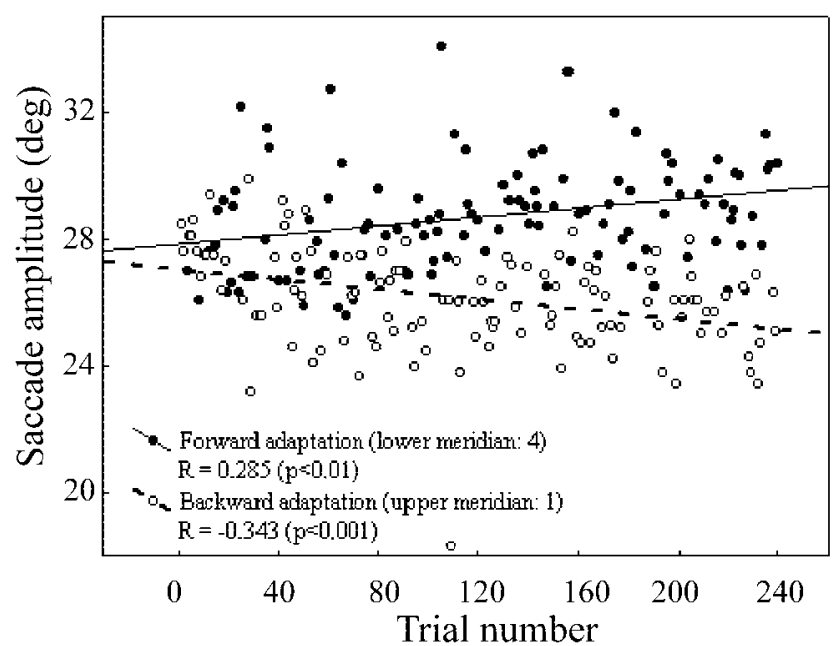

Figure 4. Simultaneous development of backward $(\bigcirc)$ and forward (O) saccadic adaptation in the BDA condition, in subject F. The amplitude of each primary saccade is plotted as a function of trial number. Linear regressions are shown separately for the backward (dashed line) and the forward (solid line) trials.

directions $(P=0.2)$ or between the two vertical eye positions (up or down; $P=0.4)$, with no significant interaction $(P=$ $0.3)$.

In conclusion, backward and forward adaptations interacted when they were performed simultaneously in a single session. For a better understanding of this interaction, we next examined the time course of adaptation.

\section{Time Course of Adaptation}

In the analyses presented so far, we investigated the saccade adaptation level based on the amplitude changes between preand posttest sessions. In this last section, we report on the changes of saccades recorded during the adaptation session, allowing us to quantify the rate of adaptation. Figure 4 illustrates the relationship between saccade amplitude and trial number for BDA in subject F. In this subject, we observed a moderate but statistically significant saccade amplitude change for the forward down-looking trials and a larger change in saccade amplitude for the backward up-looking trials. The group analysis (see Fig. 5) indicates that the sign of the linear regression slopes was always consistent with the direction of adaptation with a single exception in subject B for the UDBA condition. Furthermore, the slope differed significantly from 0 in 29 of the 32 relationships. In five subjects, all four relationships differed significantly from 0 (the identification letter of the three remaining subjects is shown in parentheses in Fig. 5). As indicated in the Methods section, we submitted the absolute slope values in these five subjects to an ANOVA with adaptation condition (BDA versus UDA) and adaptation direction (backward versus forward) as factors. No significant difference between the two adaptation conditions $(P=0.5)$ or between the adaptation directions $(P=0.2)$ was found, but a significant interaction emerged $(P<0.05)$. Post hoc tests showed that for the forward adaptation condition, the slope was smaller in the BDA condition $(0.82 \pm 0.28 \mathrm{deg} / 100$ trials $)$ than in the UDA condition $(1.48 \pm 0.35 \mathrm{deg} / 100$ trials; $P<0.05$; Fig. 5D). In contrast, for the backward adaptation condition, no such significant difference was found $(P=0.1$; Fig $5 \mathrm{C})$. Post hoc tests also revealed that in the UDA conditions, forward adaptation was significantly faster than backward adaptation (mean slope: $-0.76 \pm 0.38 \mathrm{deg} / 100$ trials; $P<0.05$, Fig. 5B), whereas, when evoked simultaneously (BDA condition), backward and for-

\section{TIME COURSE OF ADAPTATION}

Backward vs Forward

A-Bi-directional adaptation (BDA)

Figure 5. Time course of saccadic adaptation in all subjects (A-H), showing plots of the slope of the regression lines illustrated in Figure 4 for subject F. (A, B) Comparison of the time course of backward adaptation versus forward adaptation in the BDA and in the UDA condition. (C, D) Comparison of the time course of BDA versus UDA for backward and forward adaptation. An asterisk near each bar indicates that the slope differs significantly from zero ( $t$-test, $P<0.05$ ). In five subjects (identification letter without parentheses), all four relationships were statistically significant; the data of these subjects were averaged (Mean). Error bars, SD. Significance $\left({ }^{*} P<0.05\right)$ or nonsignificance (NS; $P>0.05$ ) indicated between the last two bars relate to the difference of adaptation time course between the two conditions depicted in each panel (ANOVA, see text).

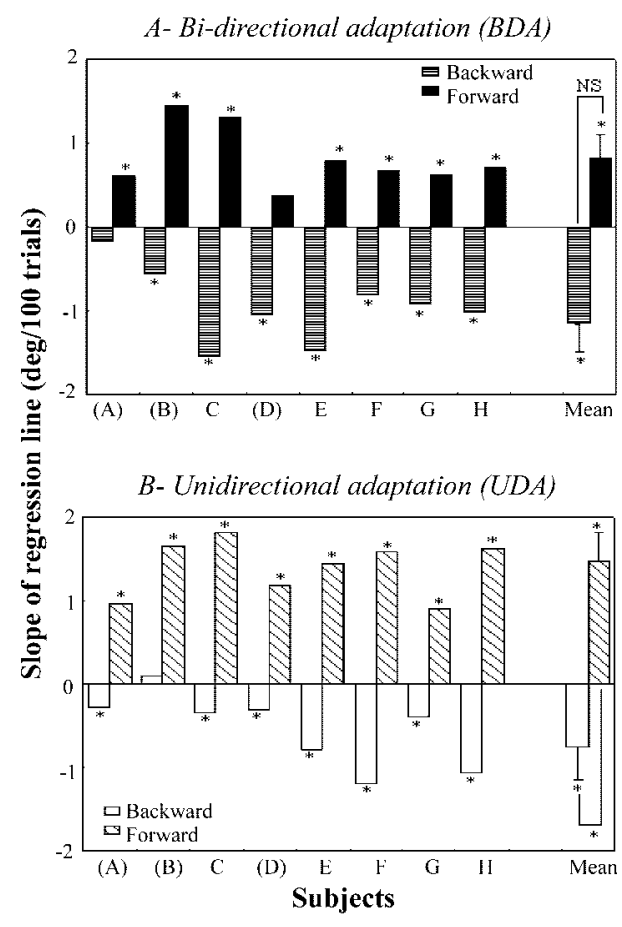

Bi- (BDA) ys Uni- (UDA) directional
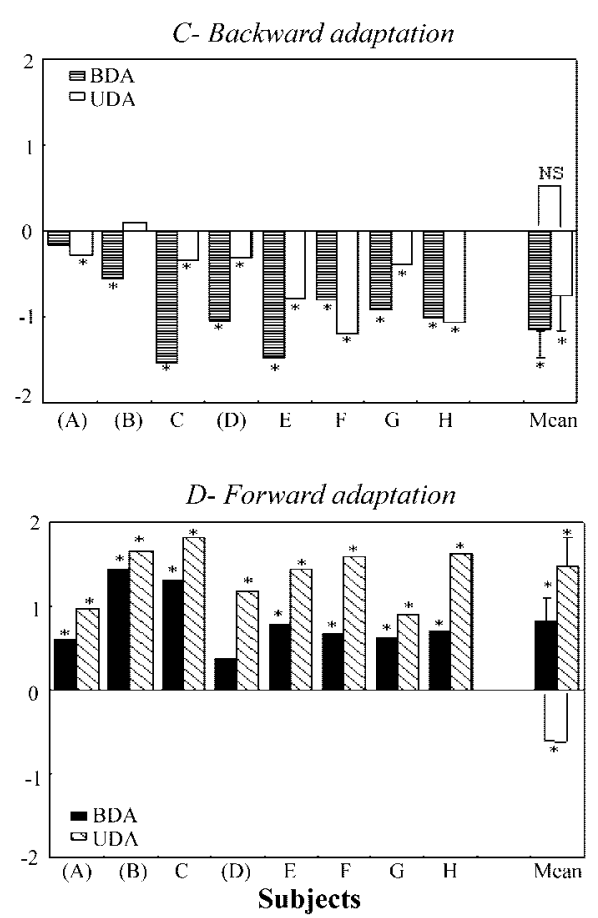
ward adaptations were equivalent in speed $(P=0.1$, Fig. 5A). The vertical eye position included as a between-subject factor in the analysis of variance again had no significant effect $(P=$ $0.6)$. In conclusion, forward adaptation was faster than backward adaptation, except when they were induced together in an interleaved fashion. In the latter case, the two opposite types of adaptation interacted with each other to the detriment of the forward adaptation. This occurred irrespective of initial orbital eye position.

\section{Discussion}

\section{Vector and Eye Position Specificity of Saccadic Adaptation}

Our purpose was to investigate whether saccadic adaptation depends only on eye displacement vector (hypothesis 1) as had been commonly implied in numerous previous studies ${ }^{2-8}$ or depends also on eye position (hypothesis 2). According to hypothesis 1, the BDA protocol would yield a strong conflict between the two opposite error signals (backward and forward). Thus, it would not be possible to induce two BDAs simultaneously (at best, one of the two adaptations would take place; at worse, none). On the contrary, if according to hypothesis 2 saccadic adaptation depends also on eye position, then the two opposite error signals would not come in conflict, so that opposite saccade amplitude changes can be obtained selectively as a function of eye position.

Our results are compatible with the predictions of hypothesis 2, indicating that saccadic adaptation is eye-position specific. Indeed, there was a significant difference between the two simultaneous modifications of saccade amplitude according to vertical eye position, suggesting that, on average, subjects were able to switch from one type of adaptation to another when varying vertical eye position. Our study brings the additional and important information that this switch operates on the short-term (i.e., every time vertical eye position changed between successive trials), because the two vertical position-dependent BDAs were interleaved on a trial-by-trial basis. In contrast, previous studies ${ }^{2-8}$ indicated that saccadic adaptation is vector specific-that is, it is linked to the direction and amplitude of the trained saccade and not to its initial or final orbital eye position. This is compatible with our study, because, in the UDA conditions, we observed a complete transfer of adaptation to nontrained saccades of the same vector as the trained saccades, but initiated from vertical eye positions intermediate between those of the two trained saccades (results not shown).

Thus, eye position appeared either essential (eye-positionspecificity hypothesis) or irrelevant (vector-specificity hypothesis). In fact, we were facing two different phenomena. In the vector-specific situation, a given saccade initiated from a particular eye position undergoes a particular type of adaptation (either backward or forward) and transfer tests evaluate to what extent the effect of that training is retained for other initial conditions (e.g., vertical eye position). In the eye-position-specific situation, two different adaptations are simultaneously called for, revealing that adaptive training can differentially affect the amplitude of saccades associated with different vertical eye positions. These two sets of data are compatible with hypothesis 2 , and we conclude: (1) that saccadic adaptation is specific to trained eye displacement and transfers to other eye positions when there is no competing training; and (2) that eye position is taken into account by the adaptive mechanisms when competing trainings are involved, each one being differentially associated with one eye position. Thus, as will be discussed below, both eye position and eye displacement signals are used by the saccade adaptive mechanisms.

\section{Limits of Eye-Position Specificity}

To determine to what extent saccadic adaptation is specific to eye position, we tested whether backward and forward adaptations in the BDA condition were as effective as in the UDA conditions (control adaptations). Our results indicate that for forward saccadic adaptation the dependence on eye position is not complete. Indeed, forward adaptation was weakened when paired with backward adaptation, compared with when it was induced alone in the control session (saccade amplitude changes: $23 \%$ vs. $65 \%$, respectively). This result illustrates the effect of a conflict with backward adaptation. This conflict appears to be partial and asymmetrical, because the backward adaptation was as efficient as when induced alone in the control session (saccade amplitude changes: $48 \%$ vs. $52 \%$, respectively). Thus, the results concerning backward adaptation are fully compatible with hypothesis 2 . An asymmetrical conflict is also consistent with the differences found in the rate of adaptation. Indeed, forward adaptation was significantly slackened when backward adaptation was performed simultaneously. This was especially noticeable, because when performed separately in the control conditions, forward adaptation was faster than backward adaptation. [Note that this last observation contrasts with previous studies showing that backward adaptation is faster and more robust than forward adaptation. $^{2,6,7,12-14}$ One possible explanation is that in our study, in which the BDA condition was always performed first, subjects may have adopted during this initial conflicting situation a strategy that favored saccade amplitude increases in the forward adaptation. This explanation, however, requires a significant retention of adaptation between the intervening period (several days), which however is not consistent with the data available so far $^{7}$ and with our unpublished observations in two human subjects. Furthermore, an influence of the order of the sessions (BDA followed by UDA) can be rejected, because the baseline measures (pretest) in the UDA conditions are identical with those of BDA conditions (see the Results section). As a general note about the analysis of adaptation time course, we add that it must be subjected to much caution because of the well-known intersubject and intrasubject variability. This variability expresses itself both in the shape of the relationship between saccade amplitude and number of trials (see the Methods section) and in the rate of adaptation, whether derived from the time-constant of exponential fitting or from the slope of linear fitting.] The asymmetrical conflict in favor of the backward adaptation suggests that forward adaptation shows less eye-position specificity. This is consistent with the previously proposed hypothesis that these two opposite types of adaptation may involve different mechanisms. ${ }^{6,12,13}$

\section{Comparison with Other Studies}

Shelhamer and Clendaniel $^{9}$ have recently investigated the effect of vertical eye position on horizontal saccadic adaptation, and their conclusions are somewhat consistent with ours. However, their paper does not provide information about the strength of BDA. Moreover, contrary to us, they did not observe any significant saccade amplitude increase with forward adaptation, but instead a slight decrease. This result could be interpreted as a complete absence of forward adaptation, corresponding to the predictions of hypothesis 1 , according to which saccadic adaptation is independent of eye position. This alternative interpretation is not readily dismissed by their control experiment. Indeed, unlike in our study, their control 
experiment involved only one of their four subjects, and they did not compare directly the efficacy with which the bidirectional adaptations develop when induced either simultaneously in a single session or independently in separate baseline conditions. A possible explanation of this difference between the two studies is that the investigators tested the two opposite types of adaptation at vertical eye positions closer to each other (elevation of $10^{\circ}$ and $-10^{\circ}$ ) than in our study, thus leading to a stronger conflict between the two opposite error signals. Another group ${ }^{14}$ has suggested that two different selective adaptations can be induced when the spatial separation between the trained saccades is only $10^{\circ}$, but their short report does not indicate the efficacy of such dual adaptations. Furthermore, both studies ${ }^{9,14}$ involved at least 600 trials compared with only 240 in the present study. Therefore, there might be a tradeoff between the number of adaptation trials and the spatial (eye position) selectivity of saccadic adaptation.

Another difference between our study and the two previous ones is that we alternated randomly across successive trials the forward and backward adaptation double-step stimuli whereas in the previous studies the stimuli were presented in blocks of 20 (or 60) forward or backward trials. Thus, our study directly demonstrates that eye position interacts with saccadic adaptation over the short-term, a situation closer to that experienced in everyday life. Because vertical eye position alternated randomly during the training as well as in the test phases, our results also indicate that eye position information is necessary both during the acquisition of adaptation and the retention of the modified behavior resulting from adaptation.

Other studies have indicated that different factors can serve as context cues for saccadic adaptation, like the target distance in depth ${ }^{15}$ and the head (roll or tilt) position. ${ }^{16}$ But here again, the efficacy of BDAs has never been evaluated in comparison to control situations in which each adaptation direction is tested separately (UDA condition). Another type of factor that can be used as context cue is the mode of saccade triggering. Indeed, all three prior studies ${ }^{11,17,18}$ that addressed the specificity of adaptation relative to that property conclude that adaptation of volitional saccades does not transfer to reflexive saccades, and vice versa. Not all factors, however, are effective as context cues. Multiple adaptive states could not be induced with cues like target color or presence or absence of background. ${ }^{19}$ One reason that these factors are not effective as context cues may be that they are not relevant for saccadic adaptation. Eye position, in contrast, is very relevant when the oculomotor system needs to adjust saccade amplitude as a result of a deficit such as an extraocular muscle weakness. ${ }^{20}$ Note that vertical eye position specificity has also been observed in the adaptation of the vestibulo-ocular reflex ${ }^{21}$ and of the smooth-pursuit eye movement ${ }^{22}$ or also for phoria adaptation. ${ }^{23}$

Concerning now the neurophysiological substrate of saccadic adaptation, much is still unknown. It is well established, however, from studies using experimental lesions in monkey $^{24-27}$ and cerebral functional imaging in humans ${ }^{28,29}$ that the cerebellum, notably the vermis and the fastigial nuclei, is involved in saccadic adaptation. In addition, these cerebellar areas receive afferent input from extraocular muscles and oculomotor commands from the brain stem (see Ref. 30 for references) which could provide both eye position and eye displacement signals necessary for saccadic adaptation. Therefore, the cerebellum is well situated to change adaptively saccade amplitude in an eye position dependent manner. Consistent with this hypothesis, previous studies of cerebellar lesions and inactivation $25,31,32$ indicated that saccadic dysmetria depends on orbital eye position, suggesting that the eye position signal is used by the cerebellum to control saccade size online. Besides, in a different sensorimotor domain, a role of the cerebellum in context-specific prism-induced adaptation of visuomanual pointing has recently been evidenced in the monkey. ${ }^{33}$ Further studies are required before this postulated role of the cerebellum can be extrapolated from monkey to humans, and from the limb sensorimotor system to the eye saccadic system.

\section{Acknowledgments}

The authors thank the subjects for their participation in the study, Marcia Riley and Christian Urquizar for designing the data replay and parameter extraction software, and Ansgar Koene for helpful remarks on the manuscript.

\section{References}

1. McLaughlin SC. Parametric adjustment in saccadic eye movements. Percept Psychophys. 1967;2:359-362.

2. Miller JM, Anstis T, Templeton WB. Saccadic plasticity: parametric adaptive control by retinal feedback. J Exp Psychol Hum Percept Perform. 1981;7:356-366.

3. Frens MA, Van Opstal AJ. Transfer of short term adaptation in human saccadic eye movements. Exp Brain Res. 1994;100:293306.

4. Deubel H. Adaptivity of gain and direction in oblique saccades. In: O'Regan JK, Levy-Schoen A, eds. Eye Movements: From Physiology to Cognition. New York: Elsevier/North-Holland; 1987:181190.

5. Albano JE. Adaptive changes in saccade amplitude: oculocentric or orbitocentric mapping? Vision Res. 1996;36:2087-2098.

6. Straube A, Fuchs AF, Usher S, Robinson FR. Characteristics of saccadic gain adaptation in rhesus macaques. $J$ Neurophysiol. 1997;77:874-895.

7. Noto CT, Watanabe S, Fuchs AF. Characteristics of simian adaptation fields produced by behavioral changes in saccade size and direction. J Neurophysiol. 1999;81:2798-2813.

8. Watanabe S, Noto CT, Fuchs A. Flexibility of saccade adaptation in the monkey: different gain states for saccades in the same direction. Exp Brain Res. 2000;130:169-176.

9. Shelhamer M, Clendaniel RA. Context-specific adaptation of saccade gain. Exp Brain Res. 2002;146:441-450.

10. Alahyane N, Pélisson D. Adaptation of saccadic eye movements: transfer and specificity. Ann NY Acad Sci. In press.

11. Deubel $H$. Separate adaptative mechanisms for the control of reactive and volitional saccadic eye movements. Vision Res. 1995; 35:3529-3540.

12. Deubel H, Wolf W, Hauske G. Adaptive gain control of saccadic eye movements. Hum Neurobiol. 1986;5:245-253.

13. Semmlow JL, Gauthier GM, Vercher JL. Mechanisms of short-term saccadic adaptation. J Exp Psychol Hum Percept Perform. 1989; 15:249-258.

14. Fujita M, Amagai A. Gaze height-dependent gain adaptation of the human horizontal saccades. Soc for Neurosci Abst. 2001.

15. Chaturvedi V, Van Gisbergen JAM. Specificity of saccadic adaptation in three-dimensional space. Vision Res. 1997;37:1367-1382.

16. Shelhamer M, Clendaniel RA. Sensory, motor, and combined contexts for context-specific adaptation of saccade gain in humans. Neurosci Lett. 2002;332:200-204.

17. Erkelens CJ, Hulleman J. Selective adaptation of internally triggered saccades made to visual targets. Exp Brain Res. 1993;93: 157-164.

18. Fujita M, Amagai A, Minakawa F, Aoki M. Selective and delay adaptation of human saccades. Cogn Brain Res. 2002;13:41-52.

19. Deubel H. Is saccade adaptation context-specific? In: Findlay JM, Walker R, Kentridge RW, eds. Eye Movement Research: Mechanisms, Processes and Applications. Amsterdam: Elsevier Science; 1995:77-187.

20. Optican LM, Zee DS, Chu FC. Adaptive response to ocular muscle weakness in human pursuit and saccadic eye movements. $J$ Neuropbysiol. 1985;54:110-122. 
21. Shelhamer M, Robinson DA, Tan HS. Context-specific adaptation of the gain of the vestibulo-ocular reflex in humans. J Vestib Res. 1992;2:89-96.

22. Takagi M, Abe H, Hasegawa S, et al. Context-specific adaptation of pursuit initiation in humans. Invest Opbthalmol Vis Sci. 2000;41: 3763-3769.

23. Schor C, Gleason G, Maxwell J, Lunn R. Spatial aspects of vertical phoria adaptation. Vision Res. 1993;33:73-84.

24. Optican LM, Robinson DA. Cerebellar-dependent adaptative control of primate saccadic system. J Neurophysiol. 1980;44:10581076.

25. Goldberg ME, Musil SY, Fitzgibbon EJ, Smith M, Olson CR. The role of the cerebellum in the control of saccadic eye movements. In: Mano N, Hamada I, DeLong MR, eds. Role of the Cerebellum and Basal Ganglia in Voluntary Movements. Amsterdam: Elsevier; 1993:203-211.

26. Takagi M, Zee DS, Tamargo, RJ. Effects of lesions of the oculomotor vermis on eye movements in primate: saccades. $J$ Neurophysiol. 1998;80:1911-1931.

27. Barash S, Melikyan A, Zhang M, Glickstein M, Thier P. Saccadic dysmetria and adaptation after lesions of the cerebellar cortex. J Neurosci. 1999;19:10931-10939.

28. Desmurget M, Pélisson D, Urquizar C, Prablanc C, Alexander GE, Grafton ST. Functional anatomy of saccadic adaptation in humans. Nat Neurosci. 1998;1:524-528.

29. Desmurget M, Pélisson D, Grethe JS, et al. Functional adaptation of reactive saccades in humans: a PET study. Exp. Brain Res. 2000; 132:243-259.

30. Pélisson D, Goffart L, Guillaume A. Control of saccadic eye movements and combined eye/head gaze shifts by the medio-posterior cerebellum. Prog Brain Res. 2003:142;69-89.

31. Ritchie L. Effects of cerebellar lesions on saccadic eye movements. J Neurophysiol. 1976;39:1246-1256.

32. Robinson FR, Straube A, Fuchs AF. Role of the caudal fastigial nucleus in saccade generation. II. Effects of muscimol inactivation. J Neurophysiol. 1993;70:1741-1758.

33. Lewis RF, Tamargo RJ. Cerebellar lesions impair context-dependent adaptation of reaching movements in primates. Exp Brain Res. 2001;138:263-267. 